\title{
EPIGAEIC ANT COMMUNITIES ALONG A SOIL MOISTURE GRADIENT IN GRAND TETON MONTANE MEADOWS
}

\author{
-

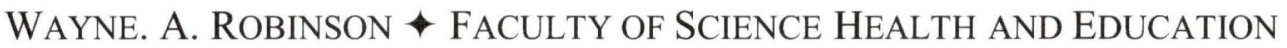 \\ UnIVERSITY OF THE SUNSHINE COAST $\downarrow$ QUEENSLAND, AUSTRALIA

\section{DiAnE. M. DEBINSKI $\downarrow$ DEPARTMENT OF ECOlOGY, EVOlution AND ORGANiSMAL BIOLOGY IOWA STATE UNIVERSITY A AMES}

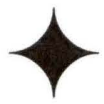

\section{$\downarrow \quad$ INTRODUCTION}

Climate change has become a major concern for scientists and resource managers across the globe. Whilst there is much speculation about the pending magnitude of the changes and their ecological effects, there is an urgent and undeniable need to have sound ecological monitoring programs in place in sensitive areas. Montane meadows in the Greater Yellowstone Ecosystem (GYE) are inhabited by short-lived plants and highly mobile animal species that can exhibit quick changes in distribution patterns relative to environmental changes (Debinski et al. 2000, Debinski et al. 2002). Thus, they can provide an early warning system for other ecosystems across the globe. Currently, the extent and range of climatic changes that will occur in montane meadows are unknown.

The biodiversity of plants, birds, and butterflies in montane meadows in the GYE have been studied annually in a long term monitoring project since 1992 (Debinski et al. 2000; Debinski et al. 1999). In these studies, meadow types were classified along a moisture gradient (from hydric to mesic to xeric). Mesic meadows have the highest magnitude of seasonal and interannual variation in aboveground photosynthetically active biomass and support the highest species diversity of plants and butterflies (Debinski et al. 2000, Debinski et al. 2002).

There is little literature on other invertebrates in montane meadow ecosystems. In this study we examine the responses of ants to the moisture gradient. Ants have been used in numerous other studies as biological indicators of environmental change (e.g. Nash et al. 1998, Agosti 2000). Furthermore because many Lycaenid butterflies can only complete their life cycle in association with particular ant species (Pierce et al. 2002, Thomas et al. 1998), it is of interest to investigate whether ant communities follow similar patterns to the plants and butterflies. Several Lycaenid butterflies specialize on these montane meadow species, including Plebejus saepiolus in the wetter meadows and Plebejus icariodes in the drier meadows (Caruthers 2008).

Ants have been described as ecosystem engineers (Jones et al. 1994) because in natural ecosystems they are important distributors of seeds (Andersen \& Morrison 1998, Auld 1999, MacMahon et al. 2003), distributors of nutrients (Eldridge \& Myers 1998, Nkem et al. 2000, MacMahon et al. 2003), and movers of soil (Folgarait 1998, Lobry de Bruyn 1999). But ants are also important and sometimes essential in the survival of many other animals including scale insects and butterflies (e.g. Pierce 1984, Seufert and Fiedler 1996). Because of their importance to ecosystem functioning and suitability for monitoring ants may be ideal indicators for monitoring ecosystem change in montane meadows from global climate change.

This first year of sampling provides a baseline for ant communities in meadows in Grand Teton National Park (GTNP). 


\section{$\downarrow \quad$ METHODS}

Sites were selected to represent a moisture gradient from hydric to xeric. The methodology of selection of sampling sites is described by Debinski et al. (2000). Six meadow types were defined, ranging from extremely hydric sedge and willow (M1) to extremely xeric (M6) sagebrush meadows. There were 25 sites located in the Teton study area (5 of each meadow type except for M4 meadows, which are not found there) including GTNP and the adjacent Teton national forest. These sites were selected to represent low elevation meadows (2000-2500 m), and specifically to avoid introducing another environmental gradient (elevation) into our analysis. This project focused solely on the 25 Teton region meadows and ant surveys were conducted during 2007.

\section{Species Characterization in Sample Sites:}

At each site a center post is permanently marked (e.g., Debinski et al. 2000). This center post is one of the corners of a $50 \times 50 \mathrm{~m}$ butterfly survey plot. The direction of the butterfly plot from the center post was randomly selected in 1997 and that location has been used annually since then. Centred in each $50 \times 50$ $\mathrm{m}$ plot we set up a $3 \times 5$ grid of points with each point $10 \mathrm{~m}$ apart. At each point a pitfall trap (Urine vials, 30 $\mathrm{mm}$ diameter, $110 \mathrm{~mm}$ depth) was opened for 7 days. Each trap was partially filled with $50 \%$ ethylene-glycol preservative.

After return to the lab the pitfall collections were sorted and all ants removed, identified and counted. Ants were initially identified to genus using Creighton (1950), then to species using Gregg (1963) and the ant collection in the Entomology museum at the University of Wyoming. A voucher collection is held by Robinson and a duplicate collection and all residuals are held at the University of Wyoming entomology museum.

Time restricted the sorting to 10 traps per site, this was usually the 10 outside traps, but on occasions when one of these traps was lost or interfered with by wildlife one of the middle traps was included. In two of the sites a majority of traps were interfered with and the complete sample set was recollected after a 1 week break. All samples were collected between $12^{\text {th }}$ June and $6^{\text {th }}$ July 2007 . Six sites were unable to be accessed because of NPS restrictions on access to these areas in the sampling period. Subsequently there are only two replicate sites for M1 and M2 categories.

\section{Species richness and diversity:}

Raw counts of ants in pitfall traps can be notoriously skewed because of positioning of the trap near nest entrances. For these data we used number of traps per site as a measure of relative abundance of each taxa at each site. We then calculated ant species richness and diversity for each site and compared the means for each measure between the 5 moisture categories using analysis of variance. The ANOVA and Student-Newman-Keuls follow up analyses have reduced power because of small and unequal sample sizes so significant effects were also investigated visually using error bar charts. For these analyses only the second set of pitfall traps were used in the sites whose initial sampled may have been affected by wildlife interference.

\section{Community Analysis:}

To determine whether there were significant affinities of ant communities between groups we performed an analysis of similarities (ANOSIM, Clarke and Warwick 1992) based on the number of traps per site for each species. Only the second set of pitfall traps was used in the two sites with repeat samples. Because there are only 2 sites in some categories we repeated the ANOSIM using low (M1+M2), medium (M3+M4) and high (M5+M6) categories for moisture. The larger samples in each category allowed pairwise follow up analyses between the categories. All ANOSIM's were based on 100,000 randomisations.

We then performed a hierarchical classification (unweighted, paired group mean average linkage strategy) of the sites using Bray-Curtis similarity. The sites were then ordinated using nonmetric multi dimensional scaling and the ant species associated with the ordination space determined using principal axis correlations. As the multivariate analyses are exploratory and not statistical we included data from the initial and repeat samples of the two sites whose data may have been corrupted in the initial sampling run.

\section{$\downarrow \quad$ Results}

10,553 ants were identified and only nine discrete species of ant were found. The ant fauna were dominated by Myrmica americanus which were present in every site and Formica individuals ( $3 \mathrm{spp}$.) Lasius sp.1 and Tapinoma sessile were moderately common, 
whilst Camponotus (2 spp.) and Solenopsis were only rarely encountered (Figure 1).

Species richness and Simspons Diversity were significantly lower in M1 (hydric) sites (Figure 2). The smaller sample sizes in M1 and M2 categories resulted in greater variability which is likely to have reduced the statistical power of the analyses. However the graph (Figure 3) reveals ant species richness and Simpson's diversity were very similar between categories M3 to M6 (mesic to xeric), and further sampling may find M2 sites intermediate in both variables.
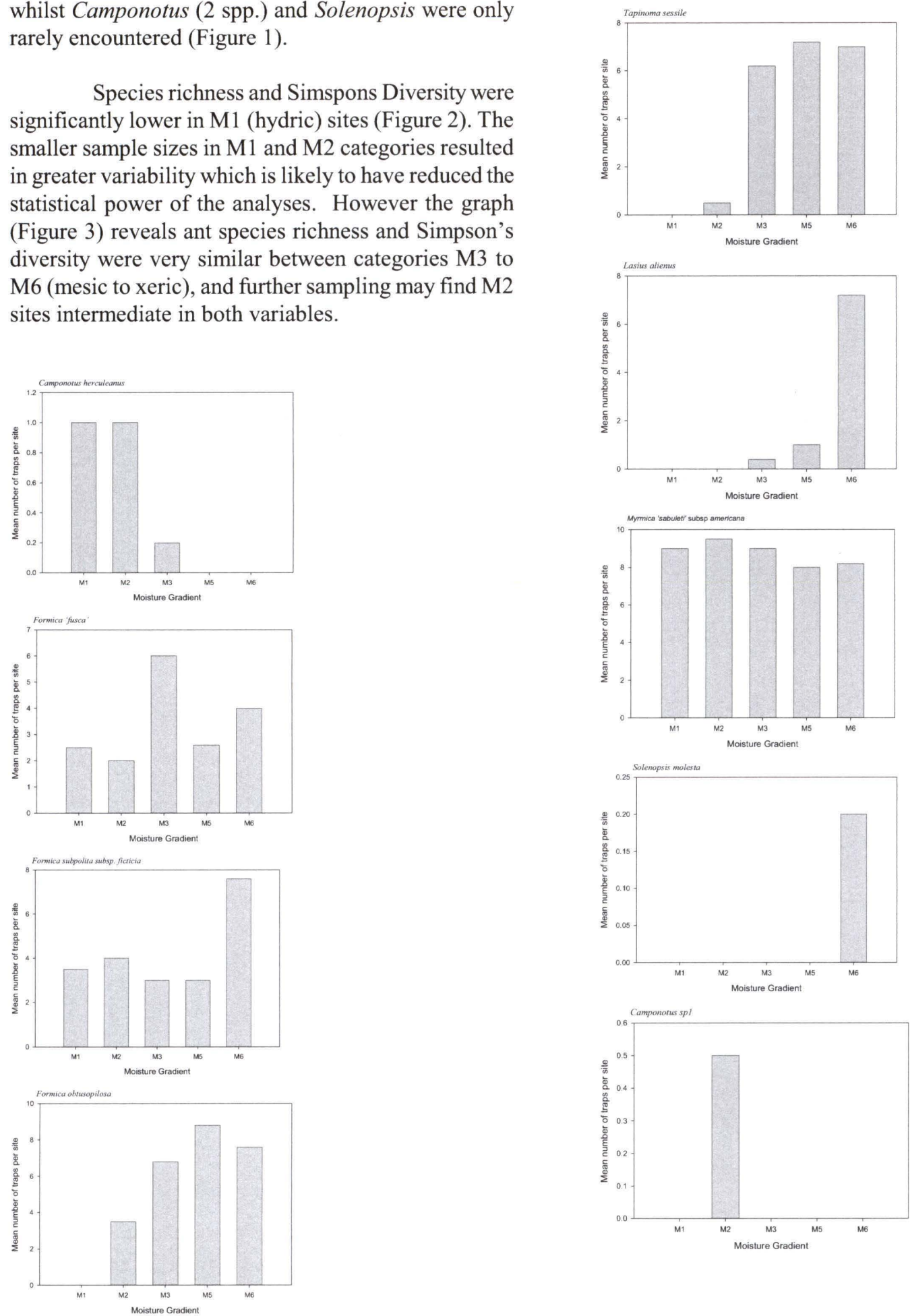

Figure 1: Relative abundance (i.e., mean number of traps in which the species was observed) of ants in 10 pitfall traps in 19 montane meadows in Grand Teton National Park and Forests. Meadows are classified from Hydric (M1) to Xeric (M6). Note that the number of replicates was 5 for M3-M6 meadows and only 2 for M1 and M2 meadows. 

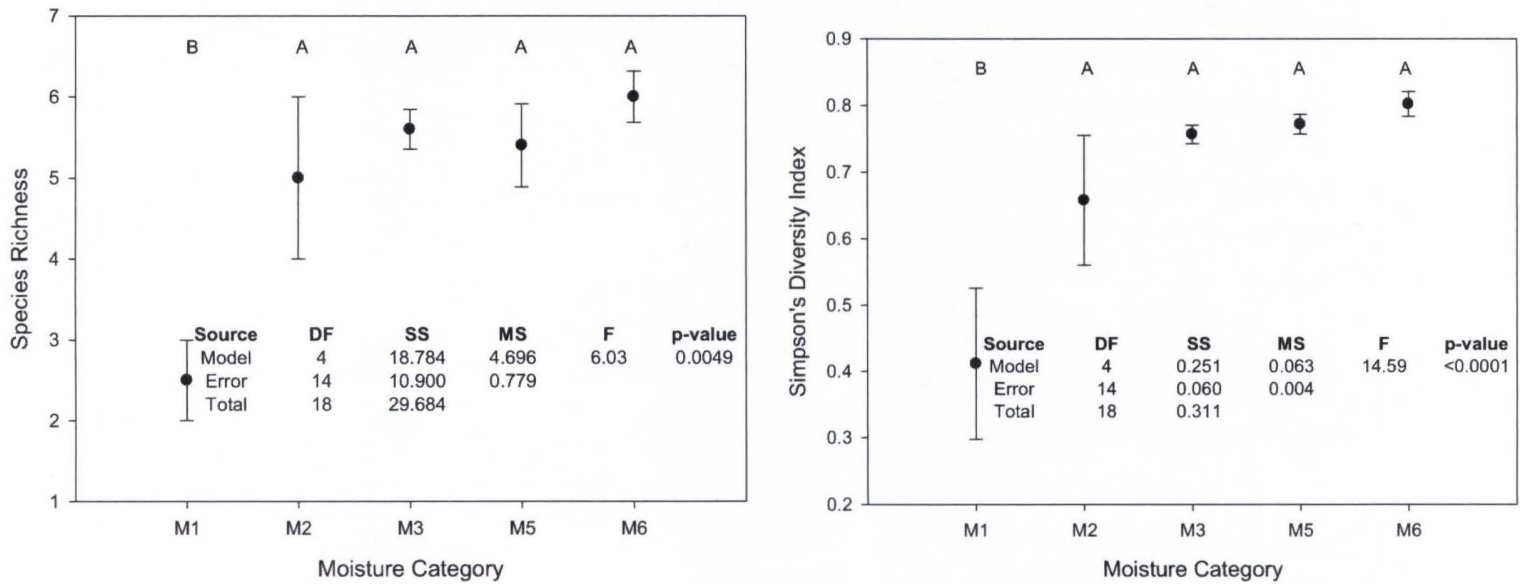

Figure 2. Ant species richness and Simpson diversity indices (Mean $+/-$ SE) in 19 sites across 5 moisture categories in GTNP in June 2007. Student-Neuman-Keul grouping is indicated by letter at top of graph.). Note that the number of replicates was 5 for M3-M6 meadows and only 2 for M1 and M2 meadows.

The analysis of similarities between the initial 5 moisture categories returned a global R statistic of 0.48 with a significance level of 0.00003 . When the data were converted to Low, Medium or High moisture categories the global $\mathrm{R}$ statistic was 0.46 with a significance level of 0.0018 . The follow up pairwise comparison found the low category was significantly different to the other two categories (Table 1). In particular there were very strong affinities $(\mathrm{R}=0.81)$ between the low and high category communities (Table

\begin{tabular}{|c|c|c|}
\hline & Medium & High \\
\hline Low & $0.51^{*}$ & $0.81^{* *}$ \\
\hline Medium & & $0.15 \mathrm{~ns}$ \\
\hline
\end{tabular}

Table 1: Pairwise ANOSIM comparisons of ant communities in low medium and high soil moisture categories in GTNP in June 2007. Values are the R statistic, ${ }^{*}=p<0.005,{ }^{* *}=p<$ 0.0005 . 1).

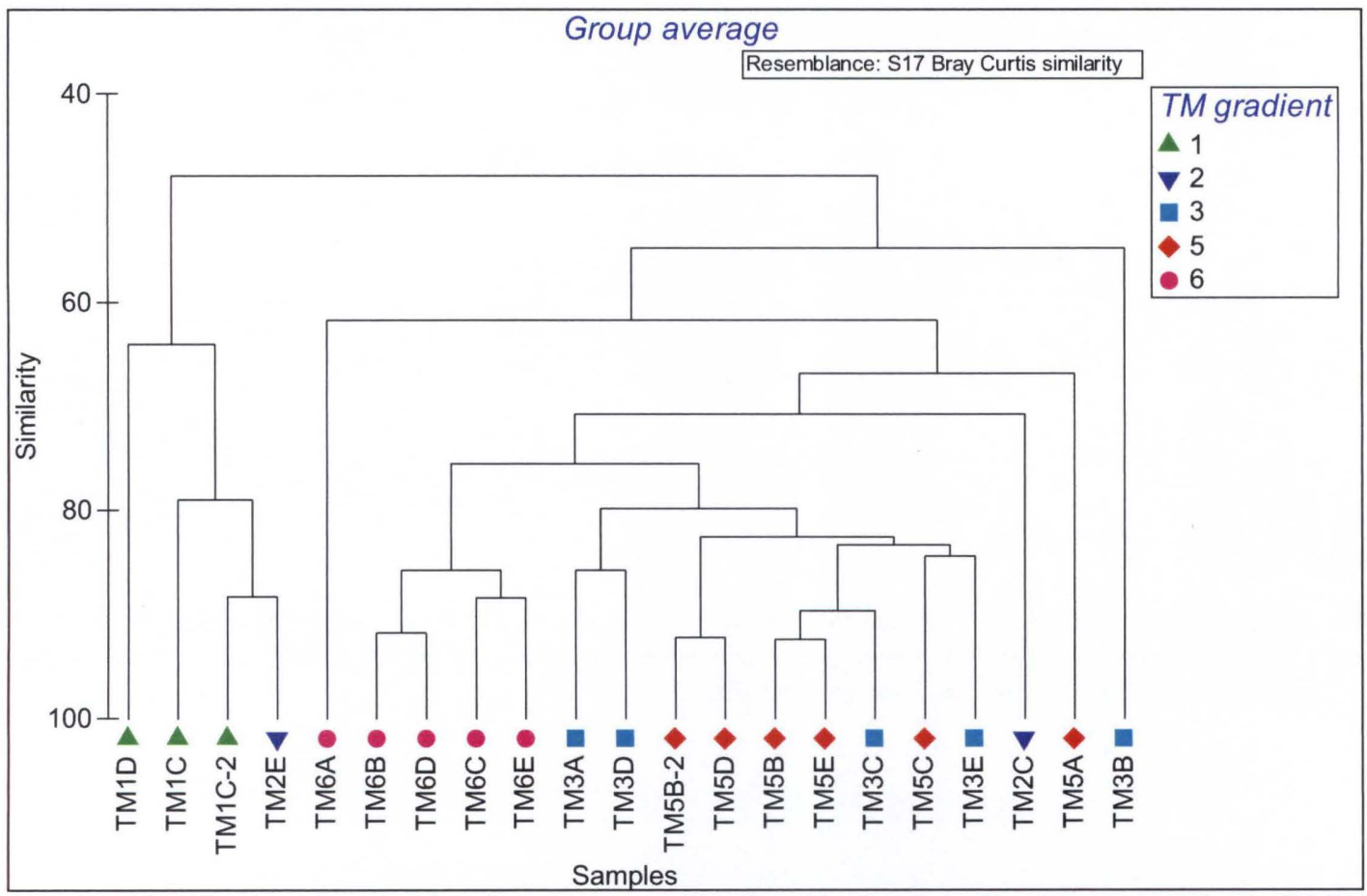

Figure 3. Classification of ground foraging ant communities found in sites from 5 moisture categories in GTNP in June 2007. Sites with labels ending in -2 are repeat samples from sites where wildlife appeared to disturb the initial samples. 
The classification shows the group of M1 sites ( + an M2 site) that are distinct and have only about $50 \%$ similarity to all the other sites (Figure 3 ). The M2 and $\mathrm{M} 3$ sites are dispersed throughout the classification suggesting intermediate stages between the M1 and M5 + M6 communities. The M6 and M5 communities are very similar with most sites showing $90 \%$ similarity between any two sites. The exceptions are site $5 \mathrm{~A}$ and $6 \mathrm{~A}$ which show $70 \%$ similarity to the others (Figure 3 ).

The ordination (Figure 4) reinforced the separation of sites as found by the classification. Camponotus herculeanus was only collected in 4 sites including two M1, one M2 and one M3 sites (Figure 4). C. herculeanus was subsequently positioned in the space near the higher moisture sites. The M5 and M6 all positioned low in MDS axis 1 and were associated strongly with Lasius alienus and Tapinoma sessile in particular. The three Formica species also tended to associate more with the xeric sites (Figure 4). The ubiquitous Myrmica americanus occurred in at least 4 traps in every site and the rare Camponotus sp 1 (1 site) and Solenopsis molesta (2 sites) were not significantly correlated with the ordination space.

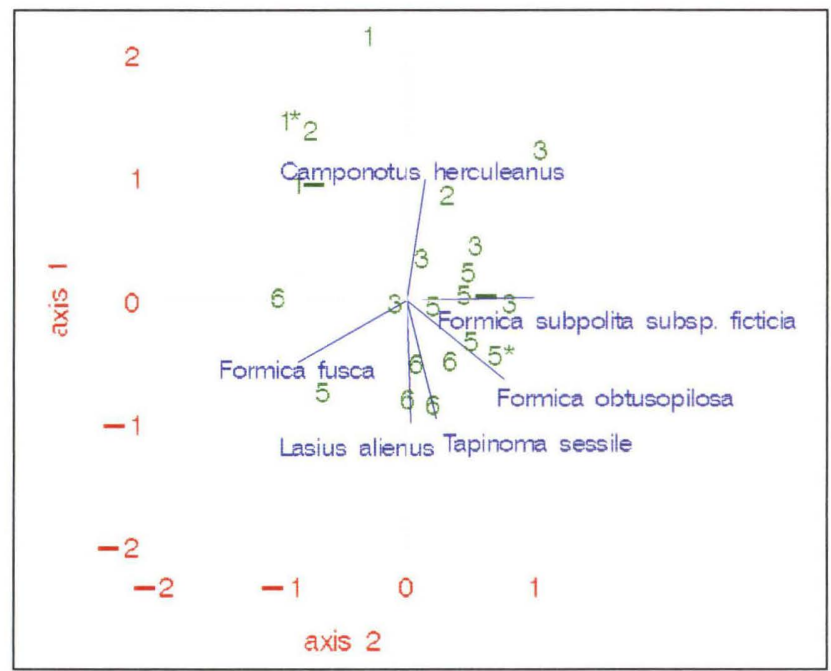

Figure 4. Ordination of ground foraging ant communities found in sites from 5 moisture categories in GTNP in June 2007. Green numbers denote meadow types. Sites with labels ending in $*$ are repeat samples from sites where wildlife appeared to disturb the initial samples. Disturbed initial samples end in -. Two dimensional stress $=0.11$. Location of species significantly correlated with the ordination space is indicated by the blue line.

\section{$\uparrow$ SUMMARY}

Overall the ant communities were able to discriminate between sites with different moisture gradients with Formica obtusopilosa and Tapinoma sessile less likely and Camponotus herculeanus more likely to occur in sites with wetter soils. Lasius alienus generally only occurred in very dry sites.

The study provides a baseline data set which should be repeated (with the missing sites) after a wetter season within the next 5 years.

\section{$\uparrow$ Literature Cited}

Agosti, D., Ed. 2000. Ants: standard methods for measuring and monitoring biodiversity. Biological diversity handbook series. Washington, D.C., 280 P. Smithsonian Institution Press.

Andersen, A.N. and S.C. Morrison. 1998. Myrmecochory in Australia's seasonal tropics - Effects of disturbance on distance dispersal. Australian Journal of Ecology 23: 483-491.

Auld, T.D. 1999. The role of ants and mammals in dispersal and post-dispersal seed predation of the shrubs Grevillea (Proteaceae). Plant Ecology 144: $201-213$.

Caruthers, J.C. 2008. Butterfly community temporal trends and responses to resource availability along a hydrologic gradient of montane meadows. M.S. Thesis, Iowa State University, Ames, IA $91 \mathrm{pp}$.

Creighton, W.S. 1950. The ants of North America. Bulletin of the museum of comparative Zoology, at Harvard College. Cosmos Press, Cambridge USA.

Debinski, D.M., M.E. Jakubauskas and K. Kindscher. 1999. A remote sensing and GIS-based model of habitats and biodiversity in the Greater Yellowstone Ecosystem. International Journal of Remote Sensing 20:3281-3292.

Debinski, D.M., M.E. Jakubauskas and K. Kindscher. 2000. Montane meadows as indicators of environmental change. Environmental Monitoring and Assessment 64:213-225. 
Debinski, D.M., M.E. Jakubauskas, K. Kindscher, E.H. Saveraid and M. Borgognone. 2002. Predicting meadow communities and species occurrences in the Greater Yellowstone Ecosystem, In: Predicting species occurrences: Issues of scale and accuracy J.M. Scott, P.J. Heglund, M. Morrison, M. Raphael, J. Haufler and B. Wall, (eds). Island Press. Covello, CA. pp. 499-506.

Eldridge, D.J. and C.A. Myers.1998. Enhancement of Soil nutrients around nest entrances of the funnel ant Aphaenogaster barbigula (Myrmicinae) in semi-arid eastern Australia. Australian Journal of Soil Research 36: 1009 1017.

Folgarait, P.J. 1998. Ant biodiversity and its relationship to ecosystem functioning: a review. Biodiversity and Conservation 7:1221-1244.

Gregg, P.S. 1963. The ants of Colorado. University of Colorado Press.

Jones, C.G., J.H. Lawton, et al. 1994. Organisms as ecosystems engineers. Oikos. 69:373-386.

Lobry de Bruyn, L.A. 1999. Ants as bioindicators of soil function in rural environments. Agricultural Ecosystems and the Environment. 74:425-441.

MacMahon, J. A., J.F. Mull, and T.O. Crist. 2003. Harvester ants (Pogonomyrmex spp.): Their Community and Ecosystem Influences. Annual Review of Ecology and Systematics 31:265 - 291.
Nash, M.S., W.G. Whitford, J. van Zee and K, Havstad. 1998. Monitoring changes in stressed ecosystems using spatial patterns of ant communities. Environmental Monitoring and Assessment. 51:201-210.

Nkem, J.N. and L.A. L. de Bruyn, et al. 2000. The impact of ant bioturbation and foraging activities on surrounding soil properties. Pedobiologia 44:609621.

Pierce, N.E. 1984. Amplified species diversity: a case study of an Australian lycaenid butterfly and its attendant ants. Symposium of the Royal Entomological Society of London 11:197-200.

Pierce, N.E., M.F. Braby, A. Heath, D.J. Lohman, J. Mathew, D.B. Rand and M.A. Travassos 2002. The ecology and evolution of ant association in the Lycaenidae (Lepidoptera). Annual Review of Entomology 47:733-771.

Thomas, J.A., D.J. Simcox, J.C. Wardlaw, G.W. Elmes, M.E. Hochberg and R.T. Clarke. 1998. Effects of latitude, altitude and climate on the habitat and conservation of the endangered butterfly Maculinea arion and its Myrmica ant hosts. Journal of Insect Conservation 2:39-46.

Seufert, P. and K. Fiedler. 1996. The influence of ants on patterns of colonization and establishment within a set of coexisting lycaenid butterflies in a South-East Asian tropical rain forest. Oecologia 106: 127-136. 